\title{
EVOLUÇÃO TEMPORAL DE FRAGMENTOS DE VEGETAÇÃO NATIVA NO MUNICÍPIO DE AGUDOS-SP, UTILIZANDO FOTOGRAFIAS AÉREAS ${ }^{1}$ \\ DÉBORA ANDRÉIA NEVES ${ }^{2}$, ZACARIAS XAVIER DE BARROS ${ }^{3}$, VERA LEX ENGEL ${ }^{4}$
}

RESUMO: A união dos processos de mapeamento aéreo e inventário florestal permitem uma análise crítica, precisa e detalhada com conclusão ágil sobre uma floresta que se deseja estudar. Seria possível saber se a área estudada se trata de um cerrado no seu sentido amplo? Este estudo tem como objetivos avaliar a evolução temporal utilizando fotografias aéreas; avaliar a ocorrência de espécies nativas lenhosas e não lenhosas utilizando levantamento florístico e fitossociológicos e, avaliar o aspecto geral da regeneração natural destes fragmentos. As duas áreas de estudo reunidas possuem aproximadamente 110 ha, parte de uma fazenda de produção de madeira localizada no município de Agudos, SP. Para elaborar a evolução temporal foram utilizadas fotografias aéreas dos anos de 1962, 1972, 1979, 2000 e 2006. E, para se conhecer a estrutura da floresta realizou-se um inventário florístico e fitossociológico. Foram demarcadas classes fitofisionômicas nos fragmentos através de uma análise aerofotogramétrica. Foram calculadas densidade, dominância e freqüência (absolutas e relativas), índice de valor de importância, índice de valor de cobertura e os índices de diversidade de Shannon, Simpson e Eqüidade. A evolução temporal mostrou as modificações sofridas pelos fragmentos no decorrer dos anos e caracterizou as áreas segundo as seis classes fitofisionômicas estipuladas. Os levantamentos florístico e fitossociológico apresentaram a condição atual dos fragmentos, ou seja, transição cerrado e floresta estacional semidecidual. Apesar da ação antrópica na região, os fragmentos se regeneram e apresentam características de recuperação. $\mathrm{O}$ uso de mapeamento aerofotogramétrico em conjunto com técnicas fitossociológicas proporcionou uma análise fisionômica da floresta e das áreas circunvizinhas, identificando a interação que existe entre ambas. Esta técnica é recomendada para o estudo de fragmentos florestais nativos.

Palavras-chave: Levantamento florístico, levantamento fitossociológico, fitofisionomia, cerrado.

\footnotetext{
${ }^{1}$ Parte da tese de doutorado do $1^{\mathrm{o}}$ autor

${ }^{2}$ Consultora Ambiental pela STCP Engenharia de Projetos - Brasil.nevesdeb@ hotmail.com

${ }^{3}$ Orientador e docente do Departamento de Engenharia Rural - FCA/UNESP -Botucatu/SP zacariasxb@fca.unesp.br

${ }^{4}$ Co-orientador e docente do Departamento de Recursos Naturais - FCA/UNESP - Botucatu/SP veralex@fca.unesp.br
} 


\section{THE FOREST THROUGH TIME EVOLUTION IN NATIVE FOREST FRAGMENTS, LO- CATED IN AGUDOS-SP, USING AIR PHOTOGRAPHS}

SUMMARY: The combination of the aerial mapping and forest inventory as processes will allow a critical, detailed and proper analysis, with agile conclusion of study object, i.e., the forest. Then, it would be possible to know if the area studied is a Savannah? Objectives: to evaluate the temporal forest evolution using air photographs; and to evaluate the occurrence of wood and not wood native species using floristic and phytosociological survey and evaluating the general aspect of the natural regeneration of these fragments. The two studied areas together are approximately $110 \mathrm{ha}$, piece of a private forest located in the city of Agudos, SP. Air photographs of the years of 1962, 1972, 1979, 2000 and 2006 were used to prepare the time evolution. A floristic and phytosociological inventory were made to know the forest structure. Phytophysiognomic class was delimited in each fragment through an aerophotogrammetry analysis. Structure, density, dominance and frequency (absolute and relative); index of importance value; index of value of covering and the diversity indices of Shannon, Simpson and Equity,were calculated with the software Fitopac 1.5 and Mata Nativa 2. Time evolution showed the modifications in the fragments through the years and characterized the areas in six determined phytophysiognomic class. The floristic and phytosociological surveys presented the current condition of the fragments, transition between Cerrado lato sensu and the seasonal semi deciduous forests. Despite of the anthropic actions in the region, forest fragments regenerate and present recovered characteristics. These results of the floristic and phytosociological surveys have been compared among themselves. The use of aerophotogrametry mapping with phytosociological techniques provided a physiognomic analysis of the forest and surrounding areas, identifying the interaction that exist between both. This technique is recommended for the study of native forest fragments.

Keywords: Floristic inventory, phytosociological inventory, phytophysiognomy, Cerrado

\section{INTRODUÇÃO}

Comunidades científicas internacionais, governos e entidades não-governamentais ambientalistas vêm alertando sobre a perda da diversidade em todo o mundo, particularmente nas regiões tropicais. A degradação biótica que está afetando o planeta encontra raízes na condição humana contemporânea, agravada pelo crescimento explosivo da população e pela distribuição desigual de riqueza. A perda da diversi- 
dade biológica envolve, desta maneira, aspectos sociais, econômicos, culturais e científicos (NEVES \& LEMOS, 2006).

O Estado de São Paulo apresenta hoje cerca de $3 \%$ de sua cobertura natural ocupada por florestas e diferentes fisionomias de cerrado, enquanto a estimativa de cobertura florestal original era de $81,8 \%$ (SÃo PAUlo, 2005). Segundo o inventário florestal da vegetação natural do estado de São Paulo (SÃo PAULO, 2005) a ocupação da vegetação natural no estado diminuiu em 36\% em um período de 27 anos (1973-2000) notadamente em áreas de cerrado, cerradão, campo cerrado e campo.

A conservação e o manejo sustentável de formações florestais naturais são dignos da atenção de uma série de processos e mecanismos que influem no funcionamento do ecossistema e de sua diversidade biológica (NEVES \& LEMOS, 2006). Assim, informações sobre dinâmica, estrutura e composição florística são elementos básicos, no que diz respeito ao Cerrado (LEMOS, 1976).

A dinâmica das florestas passa por mudanças rápidas devido a fatores que se alteram continuamente no espaço e no tempo. Fatores como a ação antrópica (adubação, melhoramento genético, dentre outros), bem como os inerentes ao clima, ao solo e a própria planta, exercem uma ação acentuada nesta dinâmica (ENGEL, 1993). Por isso, ela deve estar associada a técnicas de amostragem que sejam eficientes na denotação do estado atual e temporal da floresta, a fim de gerar informações precisas e com menor custo, para contribuir com as ações do manejo e planejamento florestal (MANTOVANI, 1990).

Segundo NAVE (1999), a necessidade de se desenvolver metodologias que permitam a análise do mosaico florestal de forma mais rápida e eficaz levou à utilização de mapeamentos aéreos. Tais mapeamentos são regularmente realizados por instituições privadas ou de capital misto podendo servir para determinar o estado da conservação e condições hierárquicas em que as florestas se encontram (PEREIRA, 1999). Desta maneira, estudos anteriormente caros, dispendiosos de tempo e com alta demanda de mãode-obra tornaram-se constantes, ágeis e com custos mais baixos possibilitando a produção de relatórios periódicos e a determinação da necessidade de intervenções de manejo na área a ser monitorada (DEGANUTTI, 2000).

A união dos processos de mapeamento aéreo e inventário florestal/levantamento fitossociológico, permitem uma análise crítica, precisa e detalhada da floresta a ser estudada, pois com a análise visual de fotografias aéreas e/ou imagens de satélite viabilizam-se soluções de complexos problemas ambientais, o que os torna imprescindível para a formação de uma base de dados. Em razão da importância desta pesquisa, deve-se fomentar o estudo dessa.

Os objetivos principais desta pesquisa estavam focados em: 
- avaliar uma evolução temporal indicando a situação dos fragmentos florestais nativos, utilizando fotografias aéreas; - avaliar a ocorrência de espécies nativas lenhosas e não lenhosas utilizando levantamento florístico e fitossociológicos; - avaliar o aspecto da regeneração natural de fragmentos florestais nativos situados dentro de plantações florestais.

\section{MATERIAL E MÉTODOS}

A fazenda Monte Alegre pertencente à empresa Duratex S.A. está localizada no km 323 da rodovia Marechal Rondon (SP-300), no município de Agudos, Estado de São Paulo, Brasil. A fazenda em questão está delimitada pelos paralelos $22^{\circ} 20^{\prime}$ e $22^{\circ} 29^{\prime}$ de latitude Sul e pelos meridianos $48^{\circ} 51^{\prime}$ e $48^{\circ} 59^{\prime}$ de longitude oeste.

Realizou-se o levantamento dos dados em duas áreas de reserva florestal constituídas por um mosaico formado por dois fragmentos de florestas nativas circundadas por reflorestamentos de Pinus spp. e Eucalyptus spp.. Tais áreas são consideradas de regeneração natural da mata nativa, constituída de poucos remanescentes da floresta estacional semidecidual e de cerrado, segundo IBGE (1992), KRONKA et al. (2003) e o Inventário florestal da vegetação natural do estado de São Paulo (SÃo PAULO, 2005) e por áreas de transição com regeneração em diferentes estágios sucessionais (PASCHOAL, 2004). O primeiro fragmento, denominado "A", tinha área de 74,21 ha e o segundo fragmento, denominado "B", tinha área de 36,25 ha.

No levantamento por fotointerpretação, a observação, demarcação das áreas e a elaboração do plano de estudo foram realizadas utilizando fotografia aérea ampliada colorida em escala nominal aproximada de 1:6.000, gerada a partir de fotografias aéreas coloridas do ano 2000, em escala nominal aproximada de 1: 30.000, bem como fotografias aéreas pancromáticas dos anos de 1962, 1972, escala 1: 25.000, 1979 escala 1:35.000 e 2000, 2006 escala 1: 30.000 para realização do estudo temporal das áreas de interesse, totalizando 12 fotografias aéreas. A fotografia aérea de 1962 não mostrava, nesta época, as fisionomias fragmentadas, como observado atualmente. Portanto, para que se pudesse realizar a evolução temporal dos fragmentos, realizou-se uma sobreposição dos mesmos, utilizando a fotografia aérea de 1972 por apresentar mesma escala (1: 25.000). 
O levantamento florístico foi realizado nos meses de abril e maio de 2005 quando se amostrou o material botânico de indivíduos herbáceo-arbustivo, arbustivo-arbóreo e arbóreo encontrados nas áreas pesquisadas. Os aspectos da regeneração natural foram analisados através de um pré-zoneamento nos níveis de altura da floresta, para se obter no mínimo, três estratos distintos e no levantamento fitossociológico utilizou o método de parcelas (GREIG-SMITH, 1983) (Figura 1). Estas parcelas foram demarcadas e são permanentes, para futuras pesquisas.

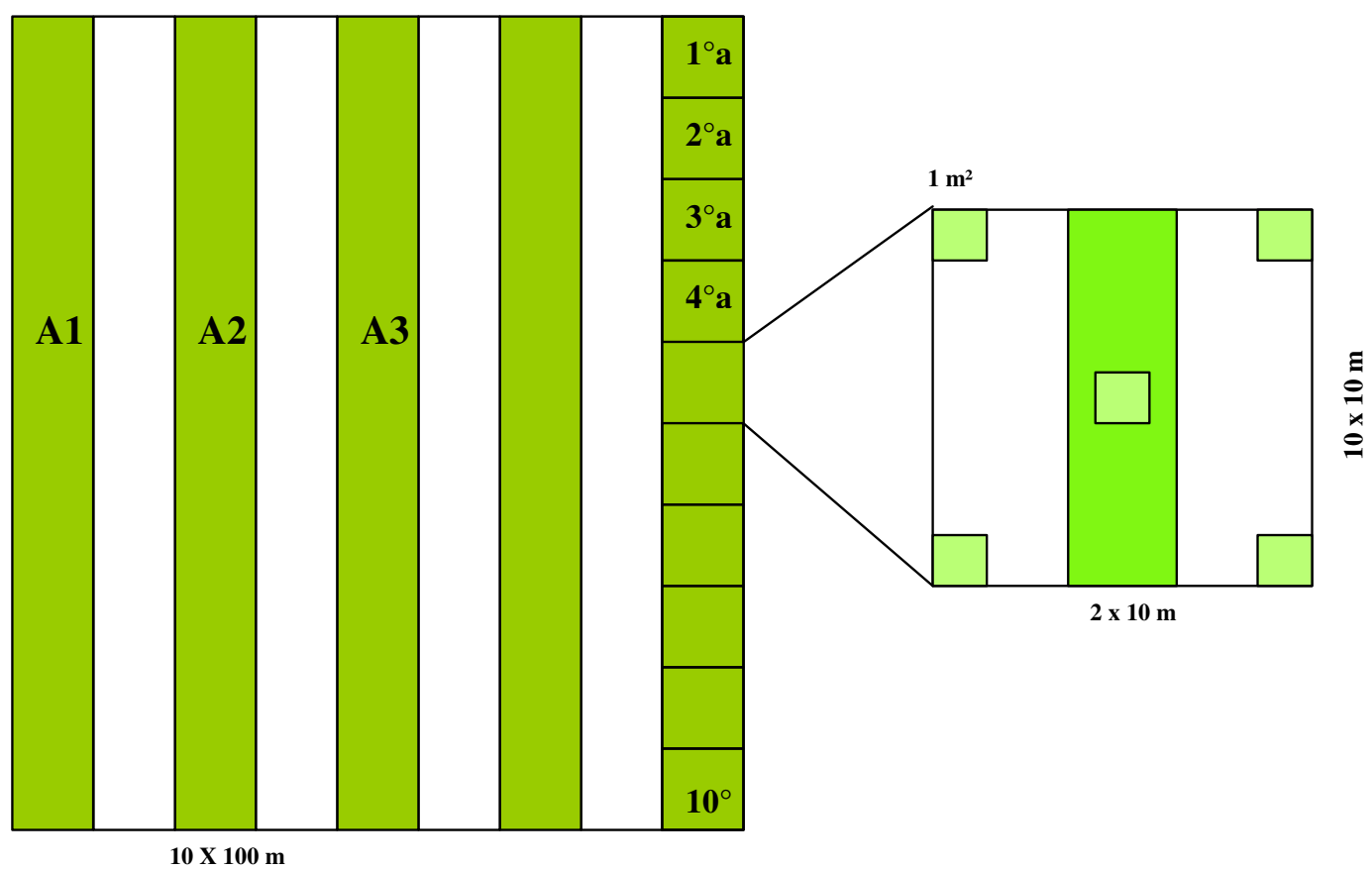

Figura 1 - Exemplo do modelo de amostragem utilizado em campo.

\section{RESULTADOS E DISCUSSÃO}

Com o resultado das análises estereoscópicas das fotografias aéreas e dos documentos cartográficos, foram identificados os seguintes geoindicadores: área urbana, solo exposto, estradas, cobertura vegetal natural, reflorestamentos com espécies exóticas e campo aberto. Após este levantamento realizou-se 
estudo da evolução temporal das áreas através da análise aerofotogramétrica, demarcando as fitofisionomias das mesmas em todas as fotografias aéreas.

Auxiliada pelos geoindicadores e baseando-se nesta evolução analisada, foram determinadas para a vegetação nativa dos fragmentos A e B, utilizando as fotografias aéreas anos de 1962, 1972, 1979, 2000 e 2006, seis classes fitofisionômicas baseadas na estrutura do dossel destes fragmentos.

Classe 1: apresentava características estruturais onde o solo estava praticamente nu, podendo apresentar algumas árvores de pequeno porte com copas de tamanho pequeno;

Classe 2: apresentava características estruturais que indicavam formações arbóreas abertas e fechadas, com dossel descontínuo, sem árvores emergentes mas, com copas de tamanhos variados;

Classe 3: apresentava características estruturais que indicavam formações arbóreas de porte pequeno, com dossel contínuo e sem árvores emergentes;

Classe 4: apresentava características estruturais que indicavam formações arbóreas de porte pequeno, com dossel contínuo podendo apresentar árvores emergentes com copas amplas;

Classe 5: apresentava características estruturais de uma floresta madura, com predomínio da fisionomia arbórea sobre as demais, com dossel descontínuo, podendo apresentar árvores emergentes com copas de tamanhos variados;

Classe 6: apresentava características estruturais de uma floresta madura, com predomínio da fisionomia arbórea sobre as demais, com dossel contínuo, podendo apresentar árvores emergentes com copas amplas;

177. Classe 7: apresentava características estruturais de estrato herbáceo e solo nu, onde as áreas de vegetação nativa foram substituídas por outras resultantes da ação antrópica. Esta classe foi elaborada para indicar os pomares de sementes implantados dentro do fragmento A, pela empresa.

Para um maior esclarecimento da evolução destas áreas tem-se a descrição e análise das classes fitofisionômicas para os fragmentos A e B, demonstradas nas fotografias da figura 2. 

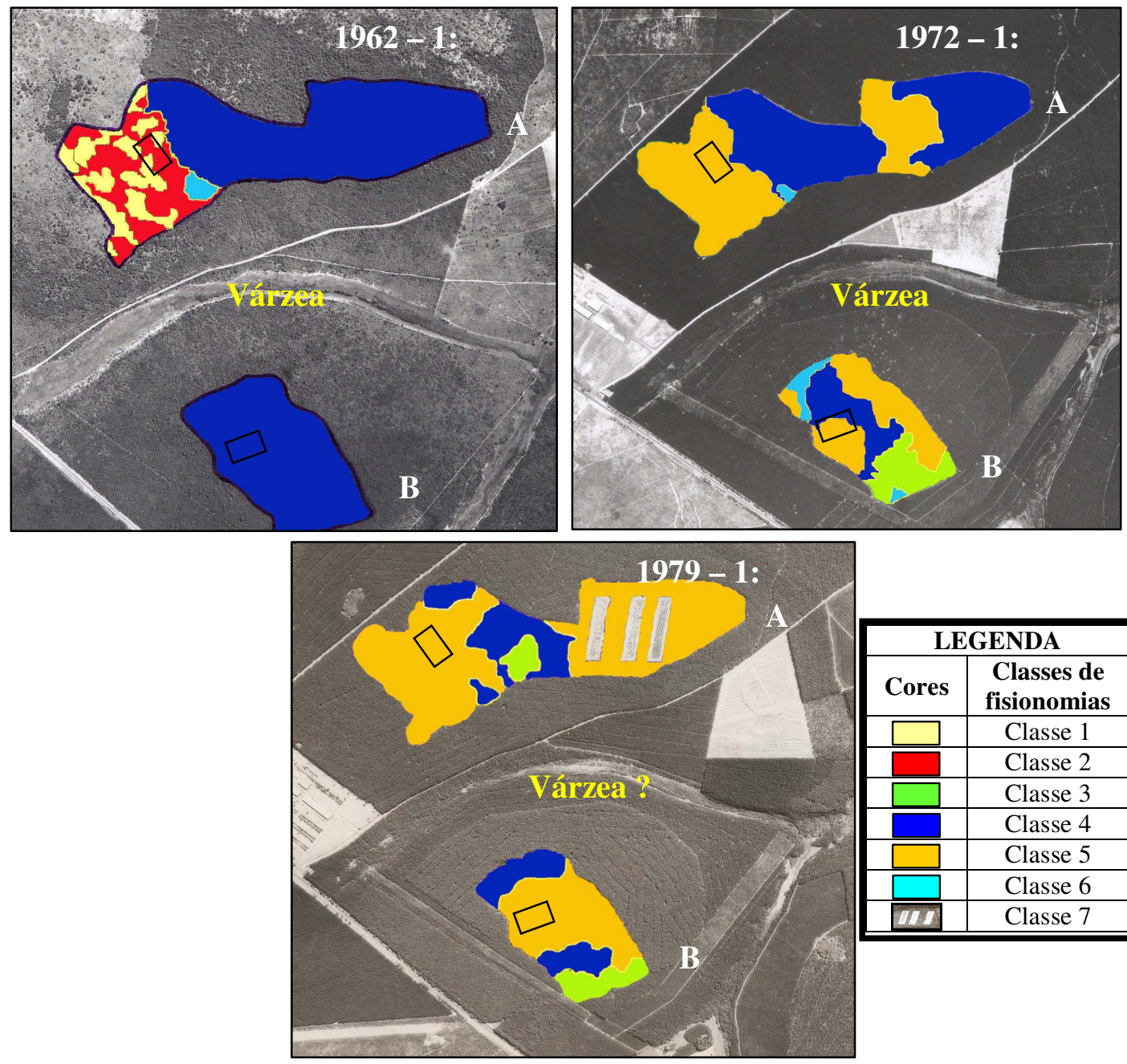

\begin{tabular}{|c|c|}
\hline \multicolumn{2}{|c|}{ LEGENDA } \\
\hline Cores & $\begin{array}{c}\text { Classes de } \\
\text { fisionomias }\end{array}$ \\
\hline$\square$ & Classe 1 \\
\hline$\square$ & Classe 2 \\
\hline$\square$ & Classe 3 \\
\hline$\square$ & Classe 4 \\
\hline$\square$ & Classe 5 \\
\hline$\square$ & Classe 6 \\
\hline$\square$ & Classe 7 \\
\hline
\end{tabular}
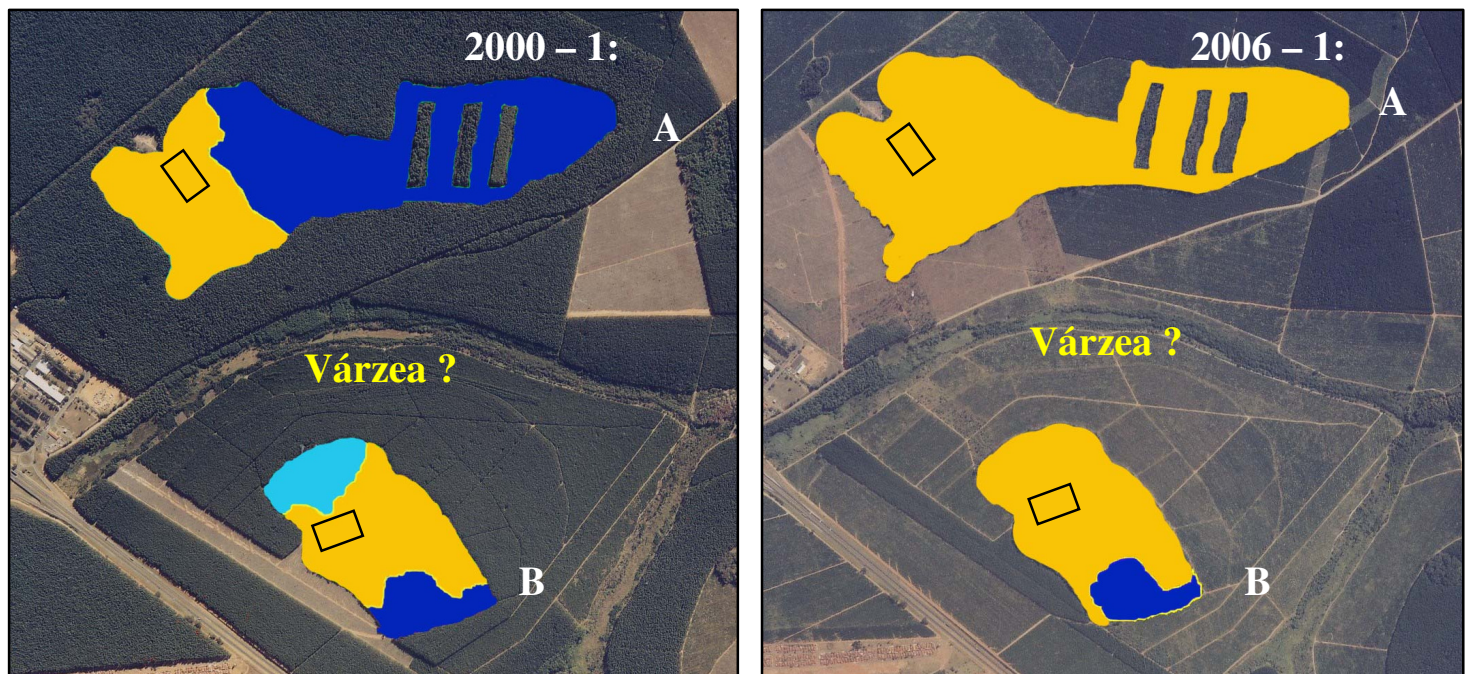

Figura 2: Histórico aerofotogramétrico dos fragmentos A e B utilizando fotografias aéreas dos anos de 1962, 1972, 1979, 2000 e 2006, com demarcações de classes fitofisionômicas. A - fragmento A; B fragmento B; Áreas de entorno - reflorestamento de Pinus spp.; $\square$ - área amostrada; ? - sem várzea. 
Os resultados do levantamento por fotointerpretação mostraram as modificações sofridas pelos dois fragmentos em cinco anos distintos. Se compararmos o fragmento A ao B, observamos que em 1962 eles aparentavam pertencer a uma mesma fisionomia. Provavelmente com a fragmentação, eles se modificaram distintamente e, no ano de 2006, voltaram a apresentar um dossel semelhante, porém com características distintas das encontradas no ano de 1962. Somente os estudos fitossociológicos realizados nas áreas pré-estabelecidas poderão concretizar o resultado demonstrado pela evolução temporal. É importante lembrar que na escolha das fisionomias visualizadas através das fotografias aéreas de todos os anos, optou-se por adotar características do dossel que fossem semelhantes aos dois fragmentos em todos os anos.

Os resultados obtidos através desta classificação fitofisionômica podem ser observados na tabela 1, onde estão demonstrados para cada ano e cada fragmento.

Tabela 1 - Situação das áreas dos fragmentos A e B em ha, nos períodos de 1962, 1972-79 e 2000-06, considerando as classes fitofisionômicas.

\begin{tabular}{|l|l|l|l|l|l|}
\hline \multirow{2}{*}{ Fragmento A } & \multicolumn{5}{|l|}{ Levantamentos (ha) } \\
\cline { 2 - 6 } & $\mathbf{1 9 6 2}$ & $\mathbf{1 9 7 2}$ & $\mathbf{1 9 7 9}$ & $\mathbf{2 0 0 0}$ & $\mathbf{2 0 0 6}$ \\
\hline Classe 1 & 16,77 & & & & \\
\hline Classe 2 & 18,49 & & & & \\
\hline Classe 3 & & & 2,53 & & \\
\hline Classe 4 & 49,89 & 44,54 & 17,84 & 50,75 & \\
\hline Classe 5 & & 30,70 & 54,86 & 24,44 & 75,23 \\
\hline Classe 6 & 1,79 & 1,0 & & & \\
\hline Classe 7 & & & 6,37 & 6,37 & 6,37 \\
\hline Total & $\mathbf{8 1 , 5 9}$ & $\mathbf{8 1 , 5 9}$ & $\mathbf{8 1 , 6 0}$ & $\mathbf{8 1 , 5 6}$ & $\mathbf{8 1 , 6 0}$ \\
\hline
\end{tabular}




\begin{tabular}{|l|l|l|l|l|l|}
\hline \multirow{2}{*}{ Fragmento B } & \multicolumn{5}{|l|}{ Levantamentos (ha) } \\
\cline { 2 - 6 } & $\mathbf{1 9 6 2}$ & $\mathbf{1 9 7 2}$ & $\mathbf{1 9 7 9}$ & $\mathbf{2 0 0 0}$ & $\mathbf{2 0 0 6}$ \\
\hline Classe 1 & & & & & \\
\hline Classe 2 & & & & & \\
\hline Classe 3 & & 7,80 & 6,1 & & \\
\hline Classe 4 & 36,25 & 10,78 & 9,28 & 6,79 & 5,40 \\
\hline Classe 5 & & 15,32 & 20,86 & 21,56 & 30,85 \\
\hline Classe 6 & & 2,4 & & 7,87 & \\
\hline Classe 7 & & & & & \\
\hline Total & & & & & \\
\hline
\end{tabular}

Levantamentos da vegetação natural do Estado de São Paulo mostram que as diferenças entre a fitofisionomias vegetacionais podem ser identificadas. Para esta identificação utilizam-se de uma legenda regional e da sua adequação àquela próxima a uma classificação de caráter universal, bem como, de procedimentos metodológicos e resultados de levantamentos da vegetação natural que antecedem o atual permitindo inferências retrospectivas para comparações e análises (SÃO PAULO, 2005). Estudos recentes utilizando a fotografia aérea para mapeamento e monitoramento de fragmentos florestais tem apresentado resultados concretos da importância da utilização desta ferramenta.

Para o levantamento florístico foram amostrado um total de 21.401 indivíduos vivos, os quais foram fornecidos resultados apenas dos indivíduos amostrados vivos. Estes indivíduos registrados pertencem a 49 famílias, distribuídos em 79 gêneros com 140 espécies, onde pelo menos 95\% destas foram identificadas até família. A espécie com maior número de indivíduos em Euphorbiaceae é o Actinostemon communis Pax \& K.Hoffm. (8.034 indivíduos), em Rubiaceae, a Psychotria sp. (1.994 indivíduos), Myrtaceae, a Eugenia sp2. (1137 indivíduos), Poaceae, a Poaceae 3 (265 indivíduos), Monimiaceae, a Siparuna guianensis Aubl. (938 indivíduos) e, em Lauraceae, a Ocotea sp. (689 indivíduos) Portanto, apenas 6 das 49 famílias continham $87,3 \%$ do número total de indivíduos amostrados.

No levantamento fitossociológico do estrato superior de regeneração para o fragmento A, com área de meio hectare, foram amostrados 7.607 indivíduos vivos pertencentes a 43 famílias, e 79 espécies. 
As espécies mais importantes foram a espécie A. communis Pax \& K.Hoffm., com 28,07\% e a espécie Pterodon emarginatus Vogel encerrando 21,41\% desta comunidade em termos de percentual de importância. Somando-se aos valores das espécies Coussarea hydrangeaefolia (Benth.) Benth. \& Hook e Xylopia aromatica (Lam.) Mart. (3,22\% e 3,09\%, respectivamente), representaram mais de 55\% da comunidade arbórea do fragmento A. No fragmento B foram amostrados 3.451 indivíduos vivos pertencentes a 27 famílias, e 63 espécies sendo as mais importantes a Faramea cyanea Müll. Arg., com 17,67\% e a Amaioua guianensis Aubl., encerrando 7,52\%, desta comunidade em termos de percentual de importância. Somando-se aos valores das espécies Copaifera langsdorffii Desf., Ocotea pulchella Mart., Psychotria sp. 2 e Siparuna guianensis Aubl. (6,1\%, 6,02\%, 5.99\% e 3,78\%, respectivamente), representaram mais de 47,1\% da comunidade arbórea do fragmento

Segundo MARTinS (1993), o elevado número de espécies com baixos valores de VI são indicadores de alta diversidade, baixa densidade das populações e da dominância. Com base nesta informação pôde-se constatar que o fragmento estudado possui uma alta densidade de populações, com a maioria das espécies possuindo valores inferiores a um. Porém a vegetação deste fragmento, ao contrário do afirmado por MARTINS (1993), possuia muitas espécies com valores baixos de VI, mas também possuia baixa diversidade.

\section{CONCLUSÕES}

A comparação dos resultados encontrados mostra que apesar da evolução temporal dos fragmentos ter apresentado classificações semelhantes para o dossel dos fragmentos, o resultado apresentado pelo levantamento fitossociológico mostrou a diferença existente entre eles. As espécies amostradas em ambos se diferenciaram em densidade, dominância, valores de importância e principalmente em valores de cobertura, mostrando que o dossel dos fragmentos é composto por espécies diferentes. Porém, apesar dos fragmentos apresentarem a composição do dossel diferentes não deixou de ter características de uma mata em transição, principalmente pelo fato da maioria destas espécies serem evidentes em várias fitofisionomias.

Utilizar fotografias aéreas para caracterização de fitofisionomias reduz bastante o tempo de pesquisa, são fáceis de trabalhar e por serem a ferramenta mais antiga do sensoriamento remoto, tem-se a possibilidade das análises temporais. Juntamente com o levantamento florístico-fitossociológico, os resultados obtidos desta união mostraram concretamente a situação da área a se pesquisar. 


\section{AGRADECIMENTOS}

À Coordenação de Aperfeiçoamento de Pessoal de Nível Superior - CAPES

\section{REFERÊNCIAS}

DEGANUTTI, R. Inventário da cobertura vegetal das fazendas Lageado e Edgardia - Botucatu-SP, no período de 36 anos, com utilização de imagens aéreas. 2000. 170 f. Tese (Doutorado em Agronomia/Energia na Agricultura)-Faculdade de Ciências Agronômicas, Universidade Estadual Paulista, Botucatu, 2000.

ENGEL, V. L. Silvigênese, dinâmica de fragmentos e a conservação de florestas tropicais. Série Técnica Florestal, Botucatu, v. 1, n. 1, 1993.

GREIG-SMITH, P. Quantitative plant ecology. 3th ed. Oxford: Blackwell Scientific, 1983. 359 p.

InSTITUTO BRASILEIRO DE GEOGRAFIA E EstatístiCA. Departamento de Recursos Naturais e Estudos Ambientais. Manual técnico da vegetação brasileira. Rio de Janeiro, 1992. 92 p.

KRONKA, F. J. N. et al.. Mapeamento de estágios sucessionais da vegetação de reserva da Copaíba e Reserva do Matão - Duratex S.A. São Paulo: Instituto Florestal, 2003. 2 p.

LEMos, A. A. B. (Org.). Cerrado: bibliografia analítica. Brasília: EMBRAPA, Departamento e Informação e Documentação, 1976. 361p.

MANTOVANI, W. A dinâmica da floresta na encosta atlântica. In: SIMPÓSIO DE ECOSSISTEMAS DA COSTA SUL E SUDESTE BRASILEIRA, 2., 1990, São Paulo. Estrutura, função e manejo: anais... São Paulo: ACIESP, 1990. v. 1, p. 304-313.

MARTINS, F. R. Estrutura de uma floresta mesófila. 2 ed Campinas: Editora UNICAMP, 1993. 245p. 
NAVE, A. G. Determinação de unidades ecológicas num fragmento de floresta nativa, com auxílio de sensoriamento remoto. 1999. 167 f. Dissertação (Mestrado em Ciências/Recursos Florestais)-Escola Superior de Agricultura Luiz de Queiroz, Universidade de São Paulo, Piracicaba, 1999.

Neves, D.A.; LEMOS, F. Biodiversidade da vegetação heerbácea em reflorestamento de Pinus sp. no Estado do Paraná - Brasil. In: PAZ GONZÁLEZ, A. Bases para la conservación de suelos y aguas em la cuenca del Río Paraná. Santa Fé: Xunta de Galicia, 2006. p. 75-86.

PASCHOAL, M. E. S. Avaliação da capacidade de regeneração da vegetação em áreas de reflorestamento com espécies de Pinus e Eucalyptus, no município de Agudos (SP). 2004. 159 f. Tese (Doutorado em Ciências Biológicas/Botânica)-Instituto de Biociências, Universidade Estadual Paulista, Botucatu, 2004.

PEREIRA, M. N.; KURKDJIAN, M. DE L. N.; ForESTI, C. Cobertura e uso da terra através de sensoriamento remoto. São José dos Campos: Instituto Nacional de Pesquisas Espaciais, 1999. 118 f. (n. 5032$\mathrm{MD} / 042)$.

SÃO PAULO (ESTADO). Secretaria do Meio Ambiente. Instituto Florestal. Inventário florestal da vegetação natural do Estado de São Paulo. São Paulo, Imprensa Oficial, 2005. 200 p. 